\title{
Le peuple en toutes lettres
}

\section{Nelly Wolf}

\section{OpenEdition}

Journals

Édition électronique

URL : http://journals.openedition.org/rhetorique/465

DOI : 10.4000/rhetorique.465

ISSN : 2270-6909

\section{Éditeur}

UGA Éditions/Université Grenoble Alpes

\section{Édition imprimée}

ISBN : 978-2-37747-084-6

\section{Référence électronique}

Nelly Wolf, «Le peuple en toutes lettres », Exercices de rhétorique [En ligne], 7 | 2016, mis en ligne le 26 mai 2016, consulté le 12 septembre 2020. URL : http://journals.openedition.org/rhetorique/465 ; DOI : https://doi.org/10.4000/rhetorique.465

Ce document a été généré automatiquement le 12 septembre 2020.

\section{(c) (i) (2)}

Les contenus de la revue Exercices de rhétorique sont mis à disposition selon les termes de la Licence Creative Commons Attribution - Pas d'Utilisation Commerciale - Partage dans les Mêmes Conditions 4.0 International. 


\title{
Le peuple en toutes lettres
}

\author{
Nelly Wolf
}

1 Le peuple n'est pas seulement ce qu'on en dit, mais ce qu'on dit de ce qu'il dit. La parole populaire est au cœur des dispositifs énonciatifs, qui dans le discours et notamment le discours littéraire, ont à charge de construire la figure du peuple. Ce corpus linguistique a été identifié il y a longtemps et des travaux récents, du côté des sciences du langage et des études littéraires, ont contribué à en renouveler l'approche. Tous ces travaux partent plus ou moins du principe que la langue du peuple est une performance orale et qu'une réflexion à son sujet engage une problématique de l'oralité. Or il semble que l'écrit participe également à la définition d'un style populaire, entendu à la fois comme ethos et comme habitus.

\section{Mimésis rédactionnelle et imaginaire de la lettre}

2 Dès le xix ${ }^{e}$ siècle, on remarque au sein de la prose narrative la présence d'écrits fictifs attribués à des personnages populaires. On constate le même phénomène au xxe siècle. On s'attardera ici sur quatre échantillons de cette mimésis rédactionnelle et on cherchera à voir si, par sa forme, sa disposition, ses effets et ses enjeux, elle se distingue de la mimésis de l'oral populaire.

1. Ne conte plus sur moi du tou, je t'embrâse pour la dernière foi. Adieu.

Louise ${ }^{1}$.

2. Élisa tes caresse sont gravé dans mon cœur. Ta bouche par ses serments leur a posé un cachet ardent. Élisa je t'aime, je t'idolatre, ma petite femme, avec un grand délire amoureux que tu as fait dissoudre dans toute ma chair. Rien au monde ne peut faire oublier tes caresse et tes baisers brulan. La mort seule me les ferai oublier. Ton amant pour la vie, pour la vie,

TANCHON, fusiller au $71^{\text {ème }}$ de ligne ${ }^{2}$.

3. Il fait froid par chez nous espérons que cela ne va pas durer. On est allés dimanche voir nos amis de Granville. La mère X est morte soixante ans ce n'est pas vieux ${ }^{3}$.

4. Moi, ma voiture plus ma femme j'avais une belle vie (Moi mas voitur+mas femme j'avais une Belle vis ${ }^{4}$. 
Les textes $n^{\circ} 1,2$ et 3 relèvent du genre épistolaire. Le premier, signé Louise, est un mot d'adieu adressé par une grisette à son amant Rodolphe. Le second, signé Tanchon, est une lettre d'amour envoyée par un soldat de ligne à sa maîtresse Élisa, une prostituée. Le troisième consiste en extraits des lettres envoyées à la narratrice par ses parents, propriétaires d'un café-épicerie dans la petite ville d'Y (Seine-Maritime). Le texte $\mathrm{n}^{\circ} 4$ relève de ce qu'on pourrait appeler un exercice parascolaire. Il a été rédigé par un détenu dans le cadre d'un atelier d'écriture en prison. L'histoire littéraire a pu attester l'authenticité documentaire partielle de la lettre de Tanchon. L'essentiel de l'extrait reproduit ici correspond au post-scriptum d'une lettre publiée dans le numéro du 17 janvier 1829 de La Gazette des tribunaux. L'auteur en est un «vieux hussard » devenu libraire. Le livre d'Ernaux se rattache à l'autofiction, celui de Bon au récit d'expérience. Ce sont des genres soumis à des exigences de véridicité, qui garantissent eux aussi, de manière quasi contractuelle, l'authenticité de leur matériel documentaire. Pourtant, les quatre textes sont des écrits fictifs parce qu'ils sont prélevés de leur contexte de communication initial pour être affectés au discours littéraire et entrer dans la logique de la représentation. On en finira avec la description de ce corpus en évoquant les modalités du discours rapporté. En fait, ces modalités se réduisent à une seule: la citation. La parole citée est partout introduite par le verbe écrire; elle est distinguée de la parole citante par des guillemets (Murger), des italiques (Bon) ou par un saut de chapitre (Goncourt) ; chez Ernaux, en revanche, l'absence de frontière visible entre les deux crée un effet d'enchâssement :

Ma mère m'écrivait un compte rendu du monde autour. Il fait froid par chez nous ${ }^{5}$ [...].

4 Venons-en maintenant à la question de l'imaginaire qui se constitue ici. L'apparition du peuple dans la sphère de l'écrit est reliée à des formes variées d'illettrisme. L'illettrisme est corrélé à la fiche d'identité socioprofessionnelle du personnage, mais, d'une certaine manière, il n'a pas besoin de cette corroboration, car l'échec dans la maîtrise du code écrit suffit à définir le personnage populaire. Un tel échec ramène les scripteurs populaires à l'oralité. Les fautes d'orthographe de Louise, de Tanchon ou du jeune détenu de Prison se rapprochent de la transcription phonétique. En supprimant les lettres non prononcées en français moderne, leur graphie aligne l'écrit sur l'oral : la dernière ${ }^{*}$ foi, tes baisers *brulan, ma *voitur... Chez Annie Ernaux, l'absence de ponctuation dans les lettres maternelles restitue le flux sonore et confie à la voix le soin de distribuer les poses accentuelles. Évaluée par divers locuteurs lettrés et relayée par des figures narratives, la performance de l'écrivant illettré est reliée à un système axiologique qui circonscrit un véritable éthos populaire. L'incorrection linguistique, la difficulté de s'exprimer correctement, le manque de familiarité avec l'univers de l'écrit ont pour corollaire un primat du cœur et du corps opposés implicitement ou explicitement à la raison et à l'intellect, privilèges des locuteurs lettrés. La présence du corps - sensible, violent, passionné, pesant ou maladroit - s'affirme et s'imprime ellemême dans l'acte d'écriture. Louise, qui était couturière avant de passer à la Bohème, " n'a guère de littérature "; " elle se borne à l'orthographe du cœur, celle qui ne met point d's au pluriel ${ }^{6} »$. Le soldat Tanchon, un ancien berger, est dit «sans lettres ${ }^{7} »$, mais sa lettre à Élisa est « une lettre écrite avec du sang », à l'exception du mot « mort $^{8}$ ». Notons que dans le document d'origine, c'est l'utilisation du sang qui constitue l'exception, puisque seul le post-scriptum bénéficie de cette technique. En barbouillant d'hémoglobine la missive de Tanchon, Edmond de Goncourt a surmotivé la métaphore de «l'encre de sang» et mis en œuvre les codes du mélodrame populaire. La lettre 
écarlate évoque le tempérament sanguin des classes inférieures qui se projette dans un ethos amoureux passionné et sentimental. Un siècle plus tard, François Bon a recours à un procédé un peu similaire. Il parle d'un texte " précisément et laborieusement écrit comme plutôt on graverait à la lame sur du bois ${ }^{9}$ ». La métaphore de l'atelier d'écriture est désactivée et réactivée par une comparaison qui, tout en introduisant un second degré figural, restitue au mot atelier son sens propre: «lieu où des artisans, des ouvriers travaillent en commun ${ }^{10} »$. S'enclenche alors un script prolétarien, où s'impose l'image d'un corps ouvrier à la peine, aux prises avec la matière et l'outil.

Ainsi semble se vérifier l'affirmation de Dominique Maingueneau : «Toute parole que la littérature donne pour "populaire" est prise dans une relation essentielle à l'oralité et au corps ${ }^{11}$. " La mention des écrits dysfonctionnels renverrait donc aux principales problématiques ouvertes par le discours littéraire lorsqu'il s'empare de la question de l'oral populaire ; le scripteur populaire ne serait qu'une variante du locuteur populaire, caractérisé par la rupture communicationnelle, l'infériorité sociale et le primat corporel.

\section{Le partage de l'écrit}

6 En fait, les deux objets, quoiqu'apparentés et souvent interconnectés, ne sont pas entièrement superposables. Pour tâcher d'y voir plus clair, on suivra brièvement les intrigues qui s'ébauchent autour de l'écrit du peuple et ses lettres illettrées.

- Hélas ! [...] La pauvre enfant n'a guère de littérature. Je suis sûr qu'elle se borne à l'orthographe du cœur, celle qui ne met point d's au pluriel. Il faudra que je lui achète un Lhomond. (SVB, 99)

Colline déclara [...] à haute voix que mademoiselle Mimi était digne de l'écharpe azurée dont on décore les impératrices du fourneau, phrase qui était complètement sanscrite pour la jeune fille, et que Rodolphe traduisait en lui disant: " Qu'elle ferait un excellent cordon bleu ». (SVB, 207)

- Vous aviez tort, Mimi : en amour, le vendredi est un bon jour; les anciens disaient : Dies Veneris. - Je ne sais pas le latin, dit mademoiselle Mimi en continuant [...]. (SVB, 331)

Le feuilletage énonciatif observable à travers ces quelques Scènes de Murger contribue à esquisser le profil linguistique du peuple et, plus particulièrement, de ses filles. Captives des commentaires métalinguistiques répartis entre différents "surlocuteurs ${ }^{12}$ ", celles-ci se définissent essentiellement par ce qui leur manque : l'orthographe, la grammaire, le latin, les figures et le vocabulaire des techniques poétiques, c'est-à-dire, un ensemble de compétences et de connaissances qui s'acquièrent dans les collèges de la Monarchie. Par contraste, le bohème apparaît comme celui qui non seulement est doté de la culture des collèges, mais la domine assez pour manifester à son égard une distance ludique en pratiquant la parodie, les calembours et le persiflage. Le narrateur malicieux réintroduit même du sens figuré dans un discours censé en être dépourvu, puisque la lettre de Louise, par un jeu de mot prêté en quelque sorte à son inconscient orthographique, convoque la métaphore du feu amoureux : « je t'embrâse... ».

8 Toutefois, cet empressement à disjoindre les territoires pourrait bien receler une inquiétude quant à leur absolue étanchéité. Les bohèmes ne sont pas très éloignés des classes pauvres, non seulement parce qu'ils en partagent souvent les lieux de résidence et la précarité, mais parce qu'ils en proviennent. Ce qui les a fait dévier de la trajectoire populaire, c'est une courte scolarisation bourgeoise. Par exemple, Murger, dont le père 
est concierge-tailleur, aurait fréquenté le collège jusqu'à l'âge de treize ans ${ }^{13}$. Alors que la poétique littéraire doit désormais se plier "aux conventions du livre ${ }^{14}$ " (Alain Vaillant), cette petite différence, réelle ou supposée, revêt une importance cruciale. Elle distingue les bohèmes du peuple sans lettre et leur donne des titres à l'écriture littéraire et à la posture artiste. Ils s'y attachent d'autant plus qu'il ne manque pas de confrères, tels les Goncourt justement, pour leur reprocher ce qu'eux-mêmes dénoncent chez leurs maitresses : pas assez de culture, pas assez de latin ${ }^{15}$.

Toutes ces précautions suffisent à peine à enrayer les risques de confusion. "La Bohème ", écrit Murger, "parle entre elle un langage particulier, emprunté aux causeries de l'atelier, au jargon des coulisses et aux discussions des bureaux de rédaction " (SVB, 42). Or dans cet idiome de la tribu, qui se donne pour "l'enfer de la rhétorique et le paradis du néologisme » (ibid.), ont migré nombre de tours familiers ou populaires : «Oh ! quéque ça me fait !...» (SVB, 145) s'écrie un critique littéraire, tandis que Rodolphe ordonne à Marcel : «A pas peur [...]» (SVB, 364). Ces emprunts à l'oralité populaire n'auraient rien de notable (on pourrait en trouver l'équivalent chez Balzac) s'ils ne rencontraient un phénomène symétrique et inverse de migration des tournures littéraires dans la langue des grisettes : «Eh bien, alors, oyez ma peine et y compatissez, noble sire, reprit Musette, qui était un peu teintée de littérature, bien qu'elle se livrât sur la grammaire à d'horribles Saint-Barthélemy [...]» (SVB, 126).

Mis bout à bout, ces énoncés construisent une situation dont Murger ne cesse de vouloir écarter l'hypothèse. Le peuple sans éducation et le peuple éduqué au collège ont bien la langue en partage. Lecteurs des mêmes feuilletons, spectateurs des mêmes mélodrames, ils sont les réalisateurs des mêmes phrases où on trouve à la fois des locutions triviales et une parodie du style médiéval affecté par le romantisme. Le mot de Louise, le vernis littéraire de Musette évoquent l'une et l'autre le bouleversement communicationnel issu de la Révolution. La scolarisation des classes populaires au XIX siècle, ponctuées par les grandes lois scolaires (Guizot en 1835, puis Ferry en 1882) s'est conclue par une démocratisation de l'écrit. Alors que les bohèmes ont pu accéder au prestige de l'écrit, ils voient ce prestige révisé à la baisse par l'arrivée dans la sphère des échanges nationaux d'une masse croissante de scripteurs populaires.

Ainsi, Murger et ses bohèmes cultivent le tout petit avantage qu'ils conservent sur les garçons et les filles issus du même milieu qu'eux : ils ont appris la grammaire dans le Lhomond, alors que les autres n'ont eu droit, au mieux, qu'aux bricolages de la deuxième grammaire. La lettre de Louise, reproduite à titre de preuve, permet de comparer les performances et de constater que, non, décidément, elles n'ont rien à voir.

\section{Scénographies spéculaires}

Le nouveau partage de la sphère écrite est anxiogène et entraîne à la fois des stratégies distinctives et des scénographies spéculaires, où l'écrit littéraire se contemple dans l'écrit populaire pour s'autodéfinir. On l'observe avec La Fille Élisa, publié vingt-six ans après les contes de la Bohème. Autour de la lettre de Tanchon s'élabore une scénographie spéculaire opposant l'agrammaticalité illettrée du scripteur populaire et l'agrammaticalité lettrée de l'écriture artiste. Nous nous permettons de renvoyer, à ce sujet, à nos travaux précédents ${ }^{16}$. 
13 Cette scénographie spéculaire, on la rencontre encore, à la fin du $\mathrm{xx}^{\mathrm{e}}$ siècle, dans le récit de François Bon. Car on a l'impression que le commentaire appliqué aux rédactions des détenus renvoie en maintes occasions au propre style du narrateur. Ainsi, à propos d'une phrase du détenu Hurlin : Le rejet est venu très tôt pour moi ( $\mathrm{Pr}, 17)$, Bon remarque « cette manière de repousser tout au bout ce qui relève du sujet et aurait dû, dans la tradition de la langue française, initier la phrase et non la conclure ». N'estce pas une torsion du même ordre qu'il impose à l'ordre syntaxique dans l'exemple suivant : «[...] puis le mot racket à telle autre absence prononcé » $(\mathrm{Pr}, 60)$, où il disloque le groupe nominal en repoussant le participe passé à la fin de la phrase et après le groupe prépositionnel? D'autres formules pourraient aussi bien relever d'une stylistique de l'auteur : l'« écluse de pronoms grammaticaux » $(\mathrm{Pr}, 55)$; « la même et si étrange manière d'évincer l'univers sujet dans le concassage du monde » (Pr, 19). Mais, à l'inverse de ce qui se passait chez Goncourt, la réflexion spéculaire des écritures prétend instaurer une équivalence et non plus une opposition hiérarchique entre les performances rédactionnelles des détenus et celle de l'écrivain auteur et signataire du livre publié.

14 La différence tient principalement au mode d'intervention du surlocuteur ${ }^{17}$. Alors que le narrateur de la Fille Élisa exclut le locuteur populaire de l'univers lettré en le renvoyant à sa condition de "sans lettres", celui de Prison introduit la phrase du peuple dans l'ordre de l'écriture lettrée en lui appliquant la glose stylistique généralement réservée au texte littéraire. On a affaire à une stratégie non plus distinctive mais égalitaire, où l'écrit du peuple, consacré par l'explication de texte, vaut ou vaudrait n'importe quelle espèce de littérature. Une telle stratégie est conforme à la posture adoptée par François Bon qui depuis Sortie d'usine (1982) consiste à redonner voix à la classe ouvrière, aux gens d'en bas, aux laissés pour compte de la mondialisation et résonne au moment de Prison avec La Misère du monde de Pierre Bourdieu parue quatre ans auparavant. Sacralisée dans son écrit, la population carcérale réincarne une figure romantique et fraternelle du peuple, celle du pauvre et du réprouvé représentant la quintessence de l'humanité. On sera attentif, à cet égard, au fait que, quand les animateurs de l'atelier transmettent des consignes d'écriture à un jeune détenu marocain, le texte joue (inconsciemment ou non) sur le sens à la fois orthographique et pénal de la faute pour susciter le thème de la rémission de peine : «[...] on lui dit que les fautes ça ne compte pas, de ne pas s'occuper des fautes [...]» (Pr, 60).

Or, le dispositif graphique adopté par François Bon perturbe ce topos romantique. En juxtaposant les versions originale et corrigée de certaines rédactions, l'une imprimée en italiques, l'autre en romaines, il montre que contrairement à l'oral incorrect, qui a rapidement été intégré aux styles littéraires modernes, la dysorthographie et l'agrammatisme ne peuvent être absorbés par le texte lettré18. Ils requièrent nécessairement une transcription et parfois une traduction, à l'intérieur même du français. Ajoutons à cela que le poète spontané qui sommeille en chaque détenu est souvent pris en défaut par le narrateur lui-même qui n'hésite pas à en dénoncer les faiblesses. Un poème imité d'Apollinaire est ainsi qualifié de «texte de frime », et ses rimes sont jugées « mauvaises » $(\mathrm{Pr}, 22)$. Il aurait pourtant fait un bon rap : « Tu étais las de marcher dans cette rue sans fin/tu étais las de voler la nuit dans cette ville sans valeur [...]» $(\mathrm{Pr}, 21)$. Ce sont donc trois postures (ou trois ethos circonstanciels, pour reprendre la distinction suggérée par Maingueneau à Meizoz $^{19}$ ) qui sont investies ici 
par le narrateur: celle du poète fraternel, qui sublime la parole du peuple; celle de l'écrivain spécialiste de la lettre, qui constate la distance qui le sépare du scripteur illettré; celle de l'animateur d'un atelier d'écriture qui évalue les travaux de ses stagiaires. À ces trois prises de positions énonciatives au sujet de l'écrit du peuple en milieu carcéral correspondent trois constructions de l'image du peuple désignant tour à tour la plénitude humaine, l'altérité sociale et la carence culturelle. Sans être incompatibles entre elles, ces images ne se complètent pas. Elles appartiennent à des traditions discursives remontant au XIX siècle, époque où les démocraties ont commencé à formaliser la figure du peuple. Il est à penser malgré tout que l'exhibition de l'illettrisme provoque un autre effet chez le lecteur contemporain que sur celui du Second Empire ou de la III $^{e}$ République. Après un siècle et demi de grammatisation des Français, et malgré plusieurs réformes de l'orthographe, la persistance massive de graphies incorrectes ou aberrantes n'a plus le statut d'un exotisme social mais celui d'une pathologie nationale que l'explosion d'Internet, en multipliant et en banalisant la circulation d'écrits agrammatisés, ne va pas tarder à rendre publique.

On s'attardera moins sur La Place d'Annie Ernaux, parce que le livre a été abondamment commenté et que sa scène discursive a été souvent explicitée par l'auteure elle-même, en texte et hors texte. Notons malgré tout que la lettre de la mère et la glose qui l'accompagne tranchent avec le reste du corpus dans la mesure où, malgré l'absence de ponctuation, l'écrit du peuple n'y est pas tant caractérisé par l'agrammatisme et la faute d'orthographe que par la performance en français élémentaire. La culture primaire de l'écrit exclut les figures et s'arrête à la dénotation : « Ma mère m'écrivait un compte rendu du monde autour. [...] Elle ne savait pas plaisanter par écrit, dans une langue et des tournures qui lui donnaient déjà de la peine. » En un sens, la narratrice fait écho au bohème de Murger. Le locuteur éduqué à l'école primaire n'accède pas aux finesses de la rhétorique. Plus important peut-être, il n'accède pas non plus à la représentation de sa propre oralité : «Écrire comme elle parlait aurait été plus difficile encore, elle n'a jamais appris à le faire » ( $\mathrm{Pl}$, ibid.). Ces quelques phrases introduisent à nouveau les éléments d'une scénographie spéculaire. Le peuple par écrit se définit autant par ses manques que par ses limites; à l'intérieur de ces limites se dessine un territoire propre: l'écrit sans effet. Au même moment, la narratrice annonce qu'elle adopte l'écriture plate: "L'écriture plate me vient naturellement, celle-là même que j'utilisais en écrivant autrefois à mes parents pour leur dire les nouvelles essentielles " $(\mathrm{Pl}, 24)$. Ce qui est en jeu, c'est donc bien la définition du style de l'auteure. Or celle-ci, en optant pour l'écriture sans effet, renonce implicitement à l'exercice fortement oralisé et polyphonique qui a prévalu dans ses trois premiers romans. Il s'agit donc de repousser la littérature oralisée dans la rhétorique ou tout du moins, dans l'artefact lettré et de dégager un modèle d'écriture indexé sur l'écrit populaire déterminé comme performance en français élémentaire. On parlera d'une stratégie non plus distinctive ni solidaire mais fusionnelle. Alors que l'écrit lettré publié ne peut absorber le texte sans orthographe ni grammaire, il peut fusionner imaginairement avec la langue écrite du peuple scolarisé, qui devient d'ailleurs ici la vraie langue maternelle de l'écrivain.

On comparera pour finir deux passages consacrés à la description du café-épicerie parental et illustrant la première et la deuxième manière d'Annie Ernaux.

Le midi, ils bagottent déjà plus et le soir, ils ont leur chouïa dans le nez. C'est avec eux que la fête commence ils rejoignent ceux qui sont restés là tout l'après-midi, les petits vieux de l'hospice, glousseurs et vicieux, les longues maladies, les accidents 
du travail aux pansements grisâtres. (Les armoires vides [1974], Paris, Gallimard, «Folio », 1984, p. 19)

Un café d'habitués, buveurs réguliers d'avant ou d'après le travail, dont la place est sacrée [...]. L'après-midi, les vieux de l'hospice libérés jusqu'à six heures, gais et bruyants, poussant la romance. Parfois, il fallait leur faire cuver rincettes et surincettes dans un bâtiment de la cour, sur une couverture, avant de les renvoyer présentables aux bonnes sœurs. $(\mathrm{Pl}, 53)$

Entre Les armoires vides et La place la transformation énonciative a entraîné une véritable refiguration du peuple. Les cabinets ont remplacé la pissotière et les petits vieux de l'hospice, vicieux et glousseurs, sont devenus juste des vieux, gais et bruyants. En réalisant enfin la rédaction d'école primaire qui lui a été autrefois interdite ( $\mathrm{Pl}, 69)$, Annie Ernaux rompt avec une tradition représentative qui héroïse, même si c'est sur le mode carnavalesque, l'altérité populaire. Dans La Place le corps grotesque et triomphant s'est effacé, remplacé par la normalité un peu triste des dominés; la saleté et la grossièreté sont devenus des habitus. Ce vocabulaire sociologique nous rappelle l'objectif assigné à l'écriture plate : soustraire l'écriture du peuple à la littérature pour l'inscrire dans la sociologie. Or l'écriture plate indexée sur la phrase élémentaire appartient à une histoire littéraire, illustrée, entre autres, par Jules Renard, CharlesLouis Philippe, Louis Guilloux ou Albert Camus. Ces références sont effacées chez Annie Ernaux. On terminera donc avec un paradoxe. L'écriture plate, opposée à l'écriture oralisée, procède en fait d'un même fantasme: saisir sans médiation esthétique l'être du peuple.

\section{NOTES}

1. Henry Murger, Préface des Scènes de la vie de bohème [1851], Paris, Gallimard, « Folio », 2001, p. 100 , désormais noté SVB.

2. Edmond de Goncourt, La Fille Élisa, Paris, G. Charpentier, 1877, p. 207-208, désormais noté FE.

3. Annie Ernaux, La Place [1983], Paris, Gallimard, « Folio », 1986, p. 89, désormais noté Pl.

4. François Bon, Prison, Paris, Verdier, 1997, p. 61, désormais noté Pr.

5. Ibid.

6. H. Murger, op.cit., p. 99.

7. E. de Goncourt, op.cit, p. 204.

8. Ibid., p. 214.

9. F. Bon, op.cit., p. 62.

10. D'après le Petit Robert.

11. "Parole populaire, Ethos discursif et roman », dans A. Petitjean \& J.-M. Privat dir., Les voix du peuple et leurs fictions, Recherches textuelles, 7, Université Paul Verlaine-Metz, 2007, p. 263-284 ; p. 263.

12. A. Rabatel, «La représentation des voix populaires dans le discours auctorial chez A.Ernaux : surénonciation et antihumanisme théorique » dans A. Petitjean \& J.-M. Privat, op.cit., p. 263-321.

13. Pour les détails biographiques, on peut consulter P. Bourdieu, Les Règles de l'art: genèse et structure du champ littéraire, Paris, Seuil, 1992, p. 111. Bourdieu suit en cela P. Martino qui affirme dans Le Roman réaliste sous le second Empire (Paris, Hachette, 1913) que Murger a fréquenté le 
collège. Mais A. Delvau (Henry Murger et la Bohème, Paris, Bachelin-Deflorenne, 1866) et E. de Mirecourt (Henry Murger, Paris, Librairie des contemporains, 1869 [ $1^{\circ}$ édition : Paris, Gustave Havard, 1856]) ne mentionnent que l'école élémentaire du quartier. Voir aussi L. Chotard : «Un "vrai" bohème : A. Léon-Noël ", dans le dossier des Scènes de la vie de Bohème, op. cit., p. 442-448.

14. A. Vaillant, L'Histoire littéraire, Paris, Armand Colin, 2010, p. 317.

15. Voir à ce sujet le Journal des Goncourt, 31 mai 1856, 18 mai 1857 et 3 février 1861 : Edmond et Jules de Goncourt, Journal : mémoires de la vie littéraire, Paris, Robert Laffont, « Bouquins », 2004, tome I, p. 176, p. 260-261 et p. 667.

16. N. Wolf, «L'oral et l'écrit », dans Corinne Grenouillet \& É. Reverzy dir., Les Voix du Peuple : XIX et $\mathrm{XX}^{e}$ siècles, actes du colloque de l'université de Strasbourg "Voix du peuple dans la littérature », Strasbourg, Presses universitaires de Strasbourg, 2006, p. 135-144.

17. Pour ce terme, voir A. Rabatel, op.cit.

18. Les essais de Queneau sont à cet égard restés sans lendemain.

19. J. Meizoz, La fabrique des singularités. Postures littéraires II, Genève, Slatkine Érudition, 2011, p. 87.

\section{AUTEUR}

\section{NELLY WOLF}

Université Charles-de-Gaulle Lille-3 